\title{
GRAINE balloon experiment in 2015
}

\section{Precise observations of cosmic gamma rays by a high-resolution emulsion telescope}

\author{
Hiroki Rokujo $^{1 \mathrm{a}}$ and GRAINE collaboration ${ }^{2}$ \\ 1 Nagoya University, Furo-cho, Chikusa-ku, Nagoya 464-8602, Japan \\ 2 Aich University of Education, ISAS/JAXA, Utsunomiya University, Okayama University of Science, Kobe University, and Nagoya \\ University, Japan
}

\begin{abstract}
Observations of cosmic gamma rays are important for studying high energy phenomena in the universe. Since 2008, the Large Area Telescope on the Fermi satellite has surveyed the whole gamma-ray sky in the sub-GeV/GeV energy region, and accumurated a large amount of data. However, observations at the low galactic latitude remains difficult because of a lack of angular resolution, increase of background flux originating from galactic diffuse gamma rays, etc. The Gamma-Ray Astro-Imager with Nuclear Emulsion (GRAINE) is a gamma-ray observation project with a new balloon-borne emulsion gamma-ray telescope. Nuclear emulsion is a high-resolution 3D tracking device. It determines the incident angle with $0.1^{\circ}$ resolution for $1 \mathrm{GeV}$ gamma rays $\left(1.0^{\circ}\right.$ for $\left.100 \mathrm{MeV}\right)$, and has linear polarization sensitivity. GRAINE aims at precise observation of gamma-ray sources, especially in the galactic plane, by repeating long-duration balloon flights with large-aperture-area $\left(10 \mathrm{~m}^{2}\right)$ high-resolution emulsion telescopes. In May 2015, we performed a balloonborne experiment in Alice Springs, Australia, in order to demonstrate the imaging performance of our telescope. The emulsion telescope that has an aperture area of $0.4 \mathrm{~m}^{2}$ was employed in this experiment. It observed the Vela pulsar (the brightest gamma-ray source in the GeV sky) at an altitude of $37 \mathrm{~km}$ for 6 hours out of the flight duration of 14 hours. In this presentation, we will report the latest results and the status of the GRAINE project.
\end{abstract}

\section{Introduction}

\subsection{Sub-GeV/GeV gamma-ray observation}

Observations of cosmic gamma rays emitted from black Holes, pulsars, super nova remnants, etc. are important to understand such high-energy objects or phenomena in the universe. AGILE [1] launched in 2007 and the Large Area Telescope on the Fermi Gamma-ray Space Telescope (Fermi-LAT) [2] launched in 2008 have surveyed the sub-GeV/GeV gamma-ray sky. They have achieved good results and contributed to the development of gammaray astronomy: detection of more than 3000 gamma-ray sources [3]; discovery of cosmic-ray proton acceleration in super nova remnants $[4,5]$.

On the other hand, new issues have come to light. Observations at the low galactic latitude remain difficult because of a lack of angular resolution, an increase of background flux originating from galactic diffuse gamma rays, etc. Unassociated sources are still included in the high rate in gamma-ray sources detected in the galactic plane [3]. Furthermore, an unexpected gamma-ray excess in the galactic center region was reported [6].

Polarimetry data of high-energy photons from pulsars etc. is important to study the mechanism of gamma-ray emission. Several observations in the hard X-ray region have been initiated, but substantially sensitive observations in the gamma ray region have not been carried out so

a e-mail: rokujo@flab.phys.nagoya-u.ac.jp far. To make more progress in gamma-ray astronomy, not only a quantitative increase in the observation data but also qualitative improvement is required.

\subsection{GRAINE project}

The Gamma-Ray Astro-Imager with Nuclear Emulsion (GRAINE) project aims at precise observations of gammaray sources by a balloon-borne gamma-ray telescope. The high-angular resolution gamma-ray telescope, called emulsion telescope, consists of nuclear emulsion films.

Nuclear emulsion is a high precision tracking detector. It can measure the incident angle and the position of charged particles with milli-radian and sub-micron resolution in spite of the thin material $\left(\sim 10^{-3}\right.$ in the radiation-length unit). Read-out process by a microscope is needed after exposure and development because of an integrating detector, but automated and fast scanning systems have been developed and used practically in accelerator neutrino experiments.

Because nuclear emulsion can determine angles of electrons and positrons at the beginning point of pair creation interaction $\left(\gamma \rightarrow \mathrm{e}^{+}+\mathrm{e}^{-}\right)$, the angular resolution for gamma rays $(10 \mathrm{MeV}-10 \mathrm{GeV})$ is about one order of magnitude higher than that of Fermi-LAT (Fig. 1).

In addition, nuclear emulsions can measure an azimuthal angle of a plane consists of electron and positron tracks, so it has sensitivity of a linear polarization of gamma rays [7]. Nuclear emulsion has no time resolution, but gamma-ray incident timing is given by a new technique 


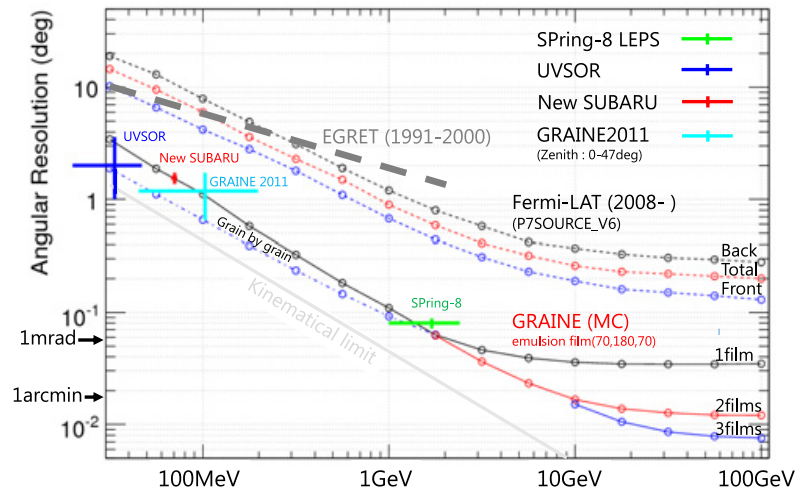

Figure 1. Angular resolution of the emulsion gamma-ray telescope (lines show simulation results and dots with error bars show experimental results), Fermi-LAT (dotted lines), and EGRET (a broken line).

Table 1. Performance of emulsion gamma-ray telescope.

\begin{tabular}{ll}
\hline Energy range & $10 \mathrm{MeV}->10 \mathrm{GeV}$ \\
\hline Angular resolution $^{1}$ & $0.93^{\circ} @ 100 \mathrm{MeV}$ \\
& $0.1^{\circ} @ 1 \mathrm{GeV}$ \\
\hline Polarization sensitivity & Yes \\
\hline Energy resolution $^{1}$ & $10 \%-20 \%$ \\
\hline Effective area $^{12}$ & $2.1 \mathrm{~m}^{2} @ 100 \mathrm{MeV}$ \\
& $2.8 \mathrm{~m}^{2} @ 1 \mathrm{GeV}$ \\
\hline FOV & $>2.2 \mathrm{sr}$ \\
\hline Time resolution & $<1 \mathrm{~s}$ \\
\hline Dead time & $\mathrm{No}$ \\
\hline
\end{tabular}

developed for GRAINE, named emulsion multi-stage shifter (described in detail in [8]).

The emulsion telescope doesn't consist of any electronic counter, so it is easy to enlarge the aperture area without deterioration of the resolution caused by limitation of the number of read-out channels. We started developing a large-area telescope (aperture area $\left.\sim 10 \mathrm{~m}^{2}\right)$ and promoting long-duration balloon flights ( $\sim 200$ hours), similar to the JACEE or RUNJOB balloonborne experiments, for scientific observation. The target objects are sources on the galactic plane/center, bright and extended sources (for example, SNR W44), galactic pulsars, gamma-ray bursts, etc. Table 1 shows the performance of the emulsion gamma-ray telescope.

\section{Balloon-borne experiment in 2015}

\subsection{GRAINE 2015}

In the first balloon experiment, GRAINE 2011, the technical feasibility was demonstrated by a small-scale emulsion telescope and a star camera system [9]. The second experiment, GRAINE 2015, aims at detecting gamma-ray sources, and demonstrating the imaging performance of the emulsion telescope in the $100 \mathrm{MeV}$ energy region. By a medium duration balloon flight (12-24 hours) in Alice Springs, Australia, observation of

\footnotetext{
${ }^{1}$ At nomal incidence.

${ }^{2}$ Effective area was considered with $10 \mathrm{~m}^{2}$ aperture area, transmittance at $5 \mathrm{~g} / \mathrm{cm}^{2}$ atmospheric depth, conversion efficiency at 0.54 radiation lengths and detection efficiency for each energy.
}

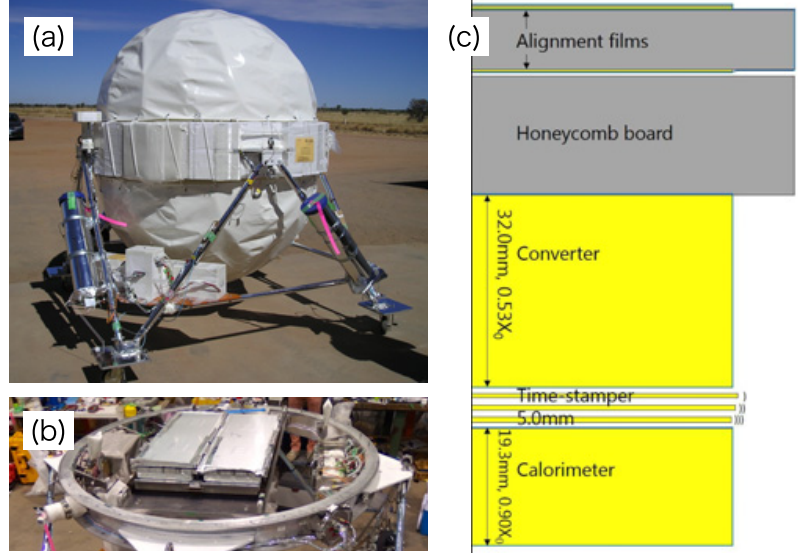

Figure 2. (a) Picture of gondola. A spherical container (the inside diameter is $1.6 \mathrm{~m}$ ) is a balloon-style pressure vessel. Three star cameras as the attitude monitors were mounted on the trusses (one of them was on the other side). (b) Inside view of the pressure vessel. At the center of a ring, the emulsion gammaray telescope was put. The total aperture area of four units was $3780 \mathrm{~cm}^{2}$. (c) Cross sectional view of the emulsion gamma-ray telescope. It consists of alignment films, the converter, and time stamper, and the calorimeter.

the Vela pulsar, the brightest gamma-ray source in the sub$\mathrm{GeV} / \mathrm{GeV}$ gamma-ray sky, for 6.5 hours (within the field of view of the emulsion telescope) is expected.

\subsection{Detector}

Figure 2 shows pictures and a cross sectional view of the emulsion gamma-ray telescope employed in GRAINE 2015. We enlarged the aperture area of the second telescope to $3780 \mathrm{~cm}^{2}$, which is 29 times larger than that of the first telescope. The converter consists of a hundred high-sensitive emulsion films. The thicknesses of the plastic layer and the both-side emulsion layers in a film are $180 \mu \mathrm{m}$ and $70 \mu \mathrm{m}$, respectively. The total thickness and the radiation length are $32 \mathrm{~mm}$ and $0.53 X_{0}$, respectively. $34 \%$ of vertical-incident gamma rays convert to electron-positron pairs, and these tracks are recorded in emulsion films. The area of a converter film is $37.8 \mathrm{~cm} \times$ $25 \mathrm{~cm}$. Four units with the same structure were employed.

An alignment unit (two or three emulsion films kept vacuum-packed with an aluminum honeycomb panel) was put on the top of each converter unit. The alignment unit is the standard surface of the detector system, and each angle of track recorded in the converter is calibrated by high momentum tracks penetrating both the alignment unit and the converter.

The multi-stage shifter system as time stamper was put at the bottom of the converter. 2-4 emulsion films were mounted on each movable stage. Three stages are driven by stepping motors. In the whole of the observation, they slide cyclically like an analog clock, and create independent combinations of the stage position. During the Vela-observation period, we changed the operation mode and made the speed of stage faster to obtain $\sim 10$ msec resolution (in the normal operation mode, the time resolution is expected to be below one second).

The energy measurement for multi-GeV gamma-ray events was performed by the analysis of the calorimeter. It has the sandwich structure of sixteen emulsion films and 


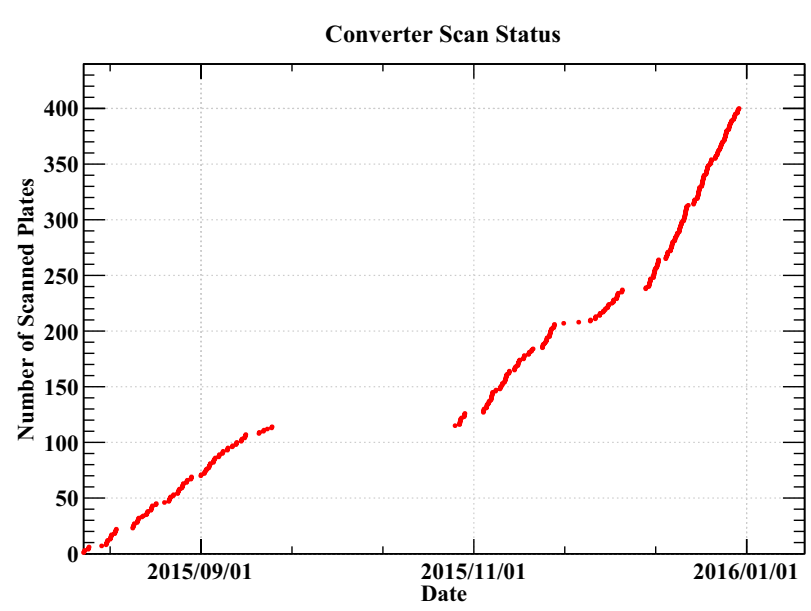

Figure 3. Performance of data taking of the converter by HTS. The vertical axis indicates the number of scanned films. The area of one film is $37.8 \mathrm{~cm}^{2} \times 25 \mathrm{~cm}^{2}$.

fifteen $1 \mathrm{~mm}$-thick stainless steel plates. The thickness and radiation length were $19.3 \mathrm{~mm}$ and $0.90 X_{0}$, respectively.

The balloon also carried three star trackers as attitude monitors, a balloon-style pressure vessel to maintain the vacuum-packed emulsion chamber, and several sensors (for GPS, temperature, pressure, etc.) as its payload.

\subsection{Balloon flight}

On May 12th, 2015, the balloon was launched from the Alice Springs balloon-launching station, Australia. The balloon left the ground at 6:33 Australian Central Standard Time (ACST), and reached $37.2 \mathrm{~km}$ altitude at 8:50. At 14:15 the Vela pulsar entered the field of view of the emulsion telescope. It observed the target for about six hours, then the balloon released the gondola at 20:22. The total flight duration in this experiment was 14.4 hours, with 11.5 hours of level flight at $36.0-37.4 \mathrm{~km}$ altitude and $4.7-3.8 \mathrm{hPa}$ residual atmospheric pressure. The gondola landed about $130 \mathrm{~km}$ north of Longreach at 20:55. On the next morning, all payloads were recovered. More detail information is described in [10].

\section{Flight data analysis}

\subsection{Data Taking by emulsion scanning system}

Data taking of emulsion films was done by the latest scanning system, Hyper Track Selector (HTS), which was developed in Nagoya University [11]. The first practical data taking of HTS went smoothly and it took about three months to finish $41 \mathrm{~m}^{2}$ of the converter films and the time stamper films. Figure 3 shows the performance of scanning by HTS.

After alignment process of segmented tracks (each track has an angle, a position, and a darkness information on a coordinate of each film), tracks running on a coordinate of the emulsion chamber were reconstructed. The track-finding efficiency of HTS was higher than $95 \%$ at $\tan \theta<2.0$ angular region as a result of evaluating the filling rate of tracks penetrating multiple films [12].

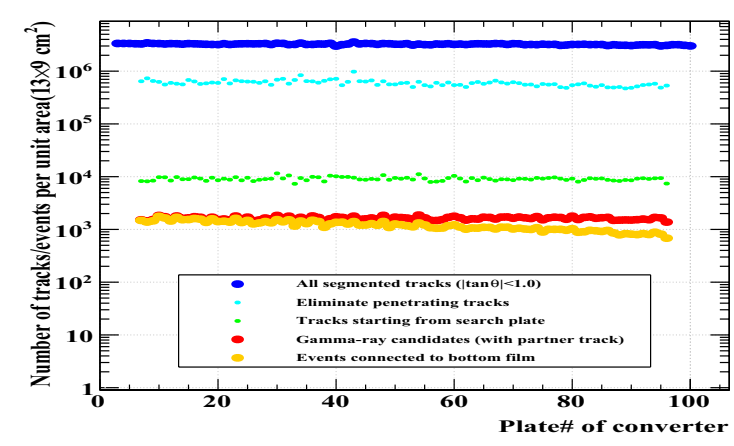

Figure 4. Result of gamma-ray event $\left(\gamma \rightarrow \mathrm{e}^{+}+\mathrm{e}^{-}\right)$selection by using a part of scan data from the converter $(13 \mathrm{~cm} \times 9 \mathrm{~cm} \times$ 100 films).

\subsection{Gamma-ray event analysis}

\subsubsection{Event detection}

Figure 4 shows a result of gamma-ray event $(\gamma \rightarrow$ $\left.\mathrm{e}^{+}+\mathrm{e}^{-}\right)$selection by using a part of scan data from the converter $(13 \mathrm{~cm} \times 9 \mathrm{~cm} \times 100$ films $) .3 \times 10^{6}$ segmented tracks at $|\tan \theta|<1.0$ angular region in each projection were scanned by HTS from a film. First, data of eight adjoining films was extracted, then tracks penetrating the volume were eliminated. Second, the following tracks were selected: tracks starting from the fourth film; tracks running to the eighth film (the bottom film in the extracted volume). This process corresponds to veto by three films of the upstream. Third, the paired topology, another independent track runs abreast nearby the track, was requested. Here, the number of tracks was reduced by $\sim 2 \times 10^{-3}$. Fourth, these electron and positron candidate tracks were connected downward from the detected film nearby the vertex, that called track follow down process. The gamma-ray event candidates which reached the bottom film (100th film of the whole of the converter unit) become the effective events for timestamp analysis. Figure 5 shows 3D views of the above processes.

\subsubsection{Energy reconstruction}

The energies of detected gamma-ray events below $\mathrm{GeV}$ were reconstructed by the converter data. The amount of multiple coulomb scattering of an electron (a positron) running in the converter was measured as RMS of angular difference and converted into momentum. Figure 6 shows a distribution of reconstructed gamma-ray energy from measured momentum of electrons and positrons. For high energy events without significant scattered angle, momentum can be determine by additional analysis of the calorimeter data.

\subsubsection{Comparison with simulation data}

We checked the response of the gamma-ray selection by Monte Carlo simulation (MC). MC data was generated by geant4.10.01. Primary gamma rays were exposed to the detector, then the response of HTS (track-finding efficiency and position and angular accuracy) were applied to electron and positron tracks. A simple power low spectrum was adopted as the energy distribution of incident gamma rays. The topological selection was performed as same as the flight data analysis mentioned above. 


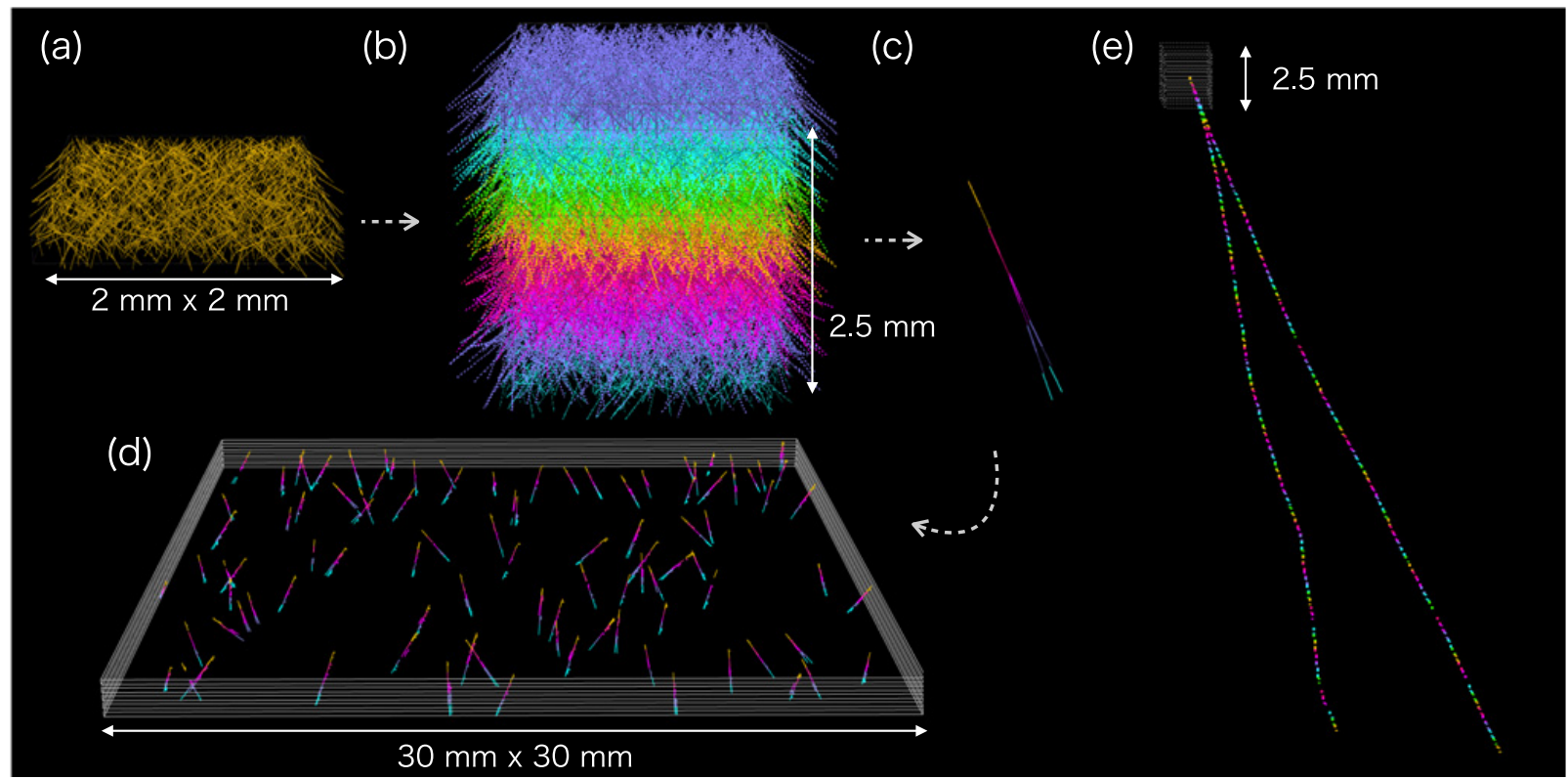

Figure 5. 3D views of scanned track data. (a) indicates $2 \mathrm{~mm} \times 2 \mathrm{~mm}$ area in a GRAINE 2015 film. the density is $\sim 400$ tracks $/ \mathrm{mm}^{2}$. (b) indicates the volume of eight films. (c) is a typical gamma-ray event detected by the selection process. (d) is a wide view ( $30 \mathrm{~mm} \times$ $30 \mathrm{~mm}$ ) as a result of gamma-ray detection. (e) shows a typical event as a result of the track follow down.

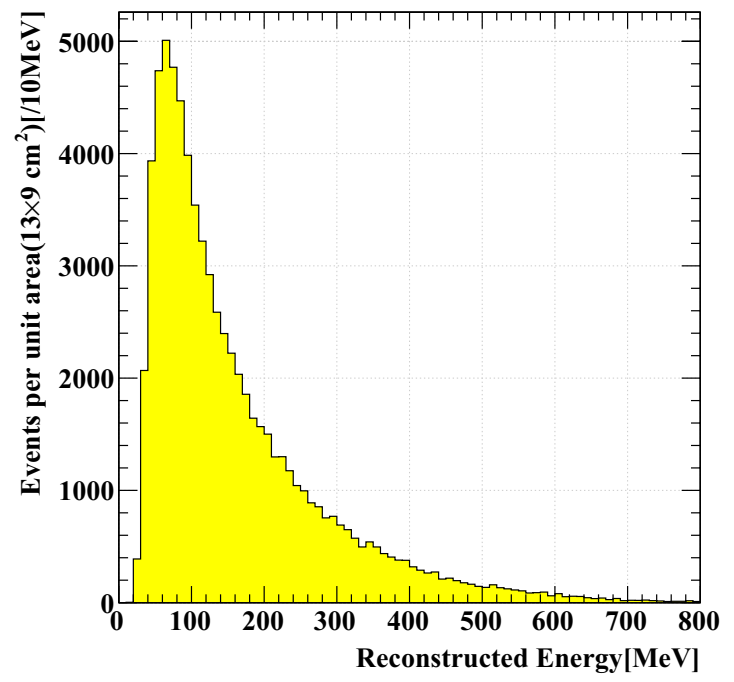

Figure 6. Distribution of reconstructed gamma-ray energy.

Figure 7 shows distributions of the opening angle between electron and positron tracks, reconstructed momentum, the ratio of the division of energy, and the invariant mass. MC data reproduced the tendencies of the flight data. In the current conventional selection, the event-pickup efficiency in the converter was estimated at $65 \%$ and $83 \%$ for $100 \mathrm{MeV}$ and $200 \mathrm{MeV}$ gamma rays, respectively.

\subsection{Background measurement at balloon altitude}

The detected gamma-ray events at the converter were connected to the time stamper, and the incident time were given to them. The main backgrounds for the observation
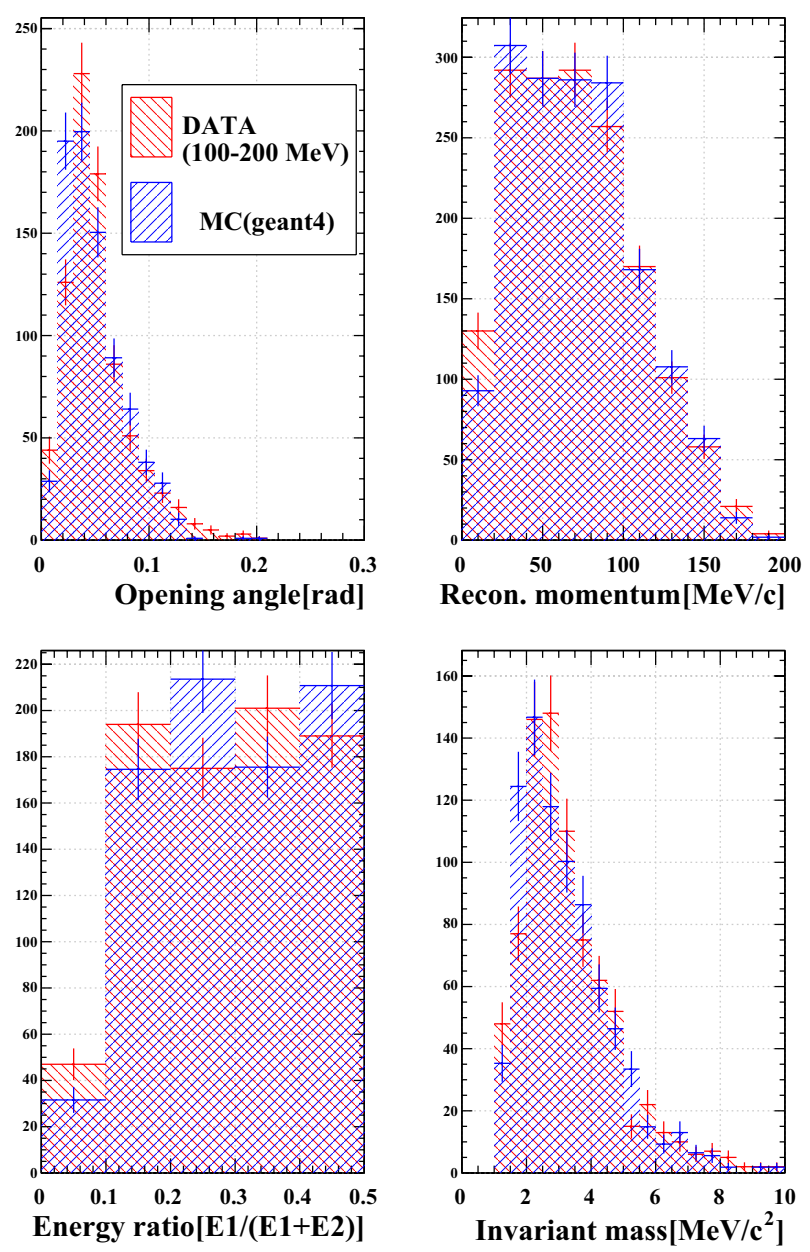

Figure 7. Comparisons of kinematical distributions between flight data and MC. 


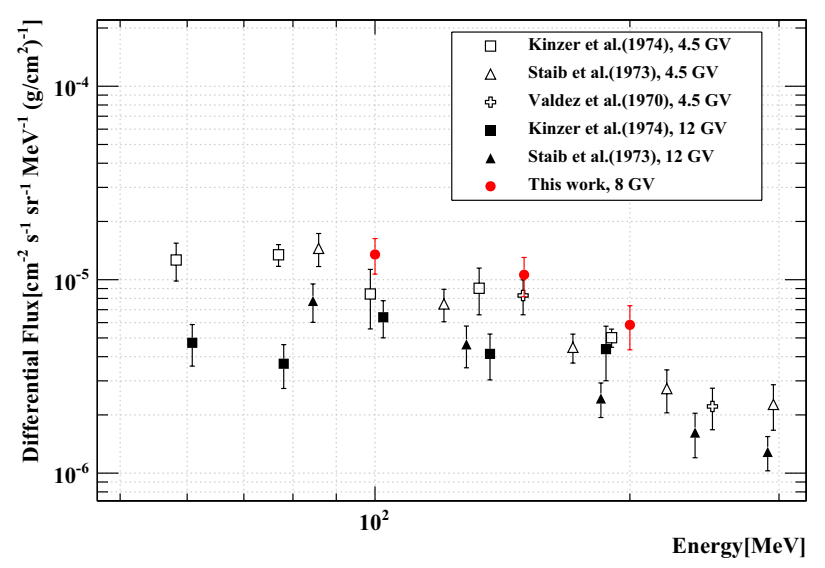

Figure 8. Preliminary result of measurement of atmospheric gamma-ray flux.

of cosmic gamma-ray sources consist of the external component (atmospheric gamma ray), and the internal component (secondary gamma ray induced in the detector by proton or electron). The latter can be rejected by identifying the hadron interaction vertex or the electron running abreast with the same incident time as the gamma ray. Here, the atmospheric gamma-ray flux was measured at the Vela-observation period (the altitude $36.0-37.4 \mathrm{~km}$ ). Gamma-ray events were selected from upstream events, which started from the 95th or upper films in the converter, to avoid the contamination of the internal component. Figure 8 shows a preliminary result of measurement of the atmospheric gamma-ray flux in $100-200 \mathrm{MeV}$ energy region. We are obtaining the tendency similar to the past measurements and studying the detector response to reduce the systematic error to $5 \%$ or less.

\section{Summary and prospects}

The second balloon experiment of GRAINE project was performed on May12th, 2015, to demonstrate the imaging performance of the emulsion gamma-ray telescope in the $100 \mathrm{MeV}$ energy region. In the experiment, a $3780 \mathrm{~cm}^{2}$ aperture telescope was employed. We succeeded in the balloon flight and recovery, and got the flight data (emulsion films). So far, $75 \%$ of data process (data taking by the scanning system, and gamma-ray event detection in the offline process) were finished. Currently, tuning of the detector response and the measurement of background is ongoing.

We will map gamma-ray events on the celestial coordinate by matching the attitude information and discuss detection of the Vela pulsar. After that, we will enlarge the aperture area and start the scientific observation with long-duration balloon flights.

The scientific balloon(DAIKIKYU) flight opportunity was provided by ISAS, JAXA. This work was supported by JSPS KAKENHI Grant Number 26247039, 26105510, and 16K17691.

\section{References}

[1] M. Tavani et al., Astron. Astrophys. 502, 995 (2009)

[2] W.B. Atwood et al., Astrophys. J. 697, 1071 (2009)

[3] F. Acero, et al., The Astrophysical Journal Supplement Series 218(2), 23 (2015)

[4] A. Giuliani, et al., The Astrophysical Journal Letters 742(2), L30 (2011)

[5] M. Ackermann, et al., Science 339(6121), 807-811 (2013)

[6] T. Daylan, et al. Physics of the Dark Universe 12, 1-23 (2016)

[7] K. Ozaki, et al., Nucl. Instrum. Meth. A 833, 165-168 (2016)

[8] S. Takahashi et al., Nucl. Instrum. Meth. A 620, 192 (2010)

[9] S. Takahashi et al., Prog. Theor. Exp. Phys. 2015, 043H01 (2015)

[10] S. Takahashi et al., Prog. Theor. Exp. Phys. 2016, 073F01 (2016)

[11] M. Yoshiomto et al., to be published in Prog. Theor. Exp. Phys.

[12] K. Ozaki et al., Journal of Instrumentation 10, P12018 (2015) 